\title{
Analysis of Pyramidal Surface Texturization of Silicon Solar Cells by Molecular Dynamics Simulations
}

\author{
Hsiao-Yen Chung, Chiun-Hsun Chen, and Hsin-Sen Chu \\ Department of Mechanical Engineering, National Chiao Tung University, Hsinchu 300, Taiwan \\ Correspondence should be addressed to Hsin-Sen Chu, hschu@cc.nctu.edu.tw
}

Received 11 April 2008; Accepted 15 May 2008

Recommended by Mohamed Sabry Abdel-Mottaleb

The purpose of this paper is to explore the relations between surface texturization and absorptance of multicrystalline silicon solar cells by a simple new model, based on the classic molecular (MD) dynamics simulation, alternative to complex electron-photon interactions to analyze the surface texturization of solar cells. In this study, the large tilted angle leads to the lower efficiency of solar cell. To consider the effect of incident angle, a range of high efficiency exists due to the increasing probability of second reflection. Furthermore, the azimuth angle of incident light also affects the efficiency of solar cells. Our results agree well with previous studies. This MD model can potentially be used to predict the efficiency promotion in any optical reflection-absorption cases.

Copyright ( $) 2008$ Hsiao-Yen Chung et al. This is an open access article distributed under the Creative Commons Attribution License, which permits unrestricted use, distribution, and reproduction in any medium, provided the original work is properly cited.

\section{INTRODUCTION}

The solar cell technology has attracted recent attention due to the price rising of petroleum. In recent studies, achieving higher efficiency in solar cells is the one of most important issues on the topic. Surface texturing of solar cells is a common approach to reduce incident light reflection and, consequently, increase solar cell efficiency.

Wet etching is commonly used to produce texturization on surface of solar cells. Generally, pyramidal structure formed on the surface of silicon solar cells by alkaline solutions etching. This pyramidal surface shape occurs because alkaline solutions etch silicon along crystallographic orientations. Hylton et al. [1] conducted many experiments to compare the efficiency increase of saw-damage etching and texture etching processes using alkaline solutions on multicrystalline silicon wafers. That paper also explained the geometrical paths of incident light which defined the absorption and reflection on the pyramid structures. Nishimoto and Namba [2] developed a low-cost wet etching manufacturing process and reported the texturization of a monocrystalline silicon surface with low cost alkaline solutions, $\mathrm{Na}_{2} \mathrm{CO}_{3}$. Recently, Gangopadhyay and his colleagues [3] further developed a new texturing method with tribasic sodium phosphate solution, claiming it was superior to the conventional method because it used less isopropyl alcohol for texturing.

New solutions are also applied. Marrero et al. [4] describe a texturization method for monocrystalline silicon solar cells based on a mixture of sodium carbonate and sodium hydrogen carbonate solutions. A specific solution has been found that results in an optimal etching rate, the lowest surface reflectance, and a homogeneous density of pyramidal structures on the silicon surface. The subsequent phosphorus diffusion with rapid thermal processes has been modified in order to drastically reduce the process time and, simultaneously, to obtain a high homogeneity of the sheet resistance values and improved photocarriers lifetimes. $100 \times 100 \mathrm{~mm}$ solar cells with an efficiency of $15.8 \%$ have been obtained compared to an efficiency of $14.7 \%$ for the reference cell.

Usual step during PV cell production is surface texturization, which is performed to reduce the reflection losses from the front surface of solar cells. Conventional chemical and electrochemical methods are efficient in texturing monocrystalline silicon [5]. Unfortunately, these methods are inefficient for multicrystalline silicon due to the presence of random crystallographic grain orientations and high 
selectivity of etching along specific directions. Consequently, it is impossible to obtain texturization of homogeneous structure on the whole surface [6-10]. Another possibility is mechanical texturization [11]. Unfortunately, it is also not satisfactory due to possible mechanical damages, resulting in breakability and fragility of silicon and additionally very small thickness of silicon wafers. Therefore, there is a real need for a versatile gentle process that is capable of producing the layer of regular texture. The possible way of overcoming these difficulties is laser texturization $[8,12,13]$.

Molecular dynamics simulation is a well-suited technique to nanoscale phenomena and mechanical behaviors [14-16]. However, few studies use MD to examine solarcell issues. Stir and Prezhdo [15] explore the photoinduced electron-transfer phenomena on a dye sensitized titanium solar cell with nonadiabatic MD (NAMD) simulations, which is based on the $a b$-initio functional theory. The system under study is typical of the dye sensitized semiconductor nanomaterials used in solar cells, photocatalysis, and photoelectrolysis. The electronic structure of the dyesemiconductor system and the adiabatic dynamics is simulated by $a b$-initio density functional theory $\mathrm{MD}$, while the NA effects are incorporated by the quantum-classical meanfield approach. A novel procedure separating the NA and adiabatic ET pathways is developed. The simulation provides a detailed picture of the ET process. For the specific system under study, ET occurs on a 30-femtosecond-time scale, in agreement with the ultrafast experimental data. Both adiabatic and NA pathways for the ET are observed. The NA transfer entirely dominates at short times and can occur due to strong localized avoided crossing as well as extended regions of weaker NA coupling. Although the adiabatic ET contribution accumulates more slowly, it approaches that of the NA ET pathway asymptotically. The electron acceptor states are formed by the d-orbital of Ti atoms of the semiconductor and are localized within the first 3-4 layers of the surface. About $20 \%$ of the acceptor state density is localized on a single $\mathrm{Ti}$ atom of the first surface layer. The simulation predicts a complex nonsingle-exponential time dependence of the ET process.

However, many unknown factors in photonelectron interactions prevent studies from being conducted on the issue of photon absorption. On the other hand, this issue is deserved to study.

The above literature review shows that there is still much necessity to investigate the problem of enhancing solar cells' efficiency. Also, the relations of surface texturization effects are still not well understood. In this study, a simple photonatomic model with molecular dynamics simulations to explore the effects solar cell surface texturization is developed and numerically solved to enhance the understanding of the underlying characteristics of different shapes of surface texturization by wet or dry etching.

The scope of this paper is mainly focused on the issue of relations of pyramidal structure and light absorptance. A real-closely pyramid texturization on the surface of multicrystalline silicon substrate etching by alkaline solutions is simulated by the molecular dynamics model to explore the influences of incident, azimuth and tilted angles with absorptance. The optimal shapes of this model are also discussed in our studies.

\section{MATHEMATICAL AND PHYSICAL MODEL}

As mentioned, the molecular dynamics simulation is a wellsuited numerical method to calculate reflection caused by texturization of solar cells. The classical molecular dynamics scheme considers an $n$-atomic nonquantum system. Each atom $i$ in this system is of its position $r_{i}$, velocity $v_{i}$ and mass $M$. Thus, the motion of a particle can be described as Newton's second law:

$$
M \frac{d^{2} r_{i}}{d t^{2}}=\sum_{j=1, j \neq i}^{N} F_{i j},
$$

where $F_{i j}$ is the force exerted by atom $j$ on atom $i$. The force term is derived from the interatomic potential that must satisfy both nanoscopic and macroscopic requirements. The potential function generally forms with a short-distance repulsive term and a long-distance attractive term. Given initial conditions, (1) can be solved to provide the position and the velocity of each particle as a function of time, which will be used to compute the system temperature, heat flux, and thermal conductivity.

Under the condition that the de Broglie wavelength of a photon and an atom is smaller, the assumption that the absorption and reflection closely represented as a series of attraction and repulsion behaviors between photons and atoms is reasonable.

To avoid complications in photonelectron transformation, a rough and simple MD model was devised to govern this problem. In the classic mechanical MD simulations, a two-body attractive-repulsive potential model governs the interaction between two particles. For one particle, shortrange repulsive forces rebound the too close particles from themselves, and long-range attraction catches other particles closer to itself in attractive force field. Assuming that the force field between an atom and photon is spherical, the 12-6 Lennard-Jones potential model with an undetermined parameter $k$, which is a short-range multiple, is adopted:

$$
U(r)=4\left[k\left(\frac{1}{r}\right)^{12}-\left(\frac{1}{r}\right)^{6}\right] .
$$

When photons reach the substrate with the nondimensional light speed $c^{*}$ and the impact angle $\theta$, attraction and repulsion behaviors occur between silicon's atoms and photons. Figure 1 shows the mechanism of photonatom interactions. When a photon enters the attractive field of an atom, the photon should be attracted to the center of atom. But the repulsive force pushes the photon off center if the photon is too close to the atom center.

The substrate is maintained as an NVE model (fixing particle numbers, system's volume and total energy) in the heat-transfer process, so the photons which enter the substrate are constrained because of forces among atoms and decay of motive energy of themselves. The system's temperature can be readily calculated from the velocity of 


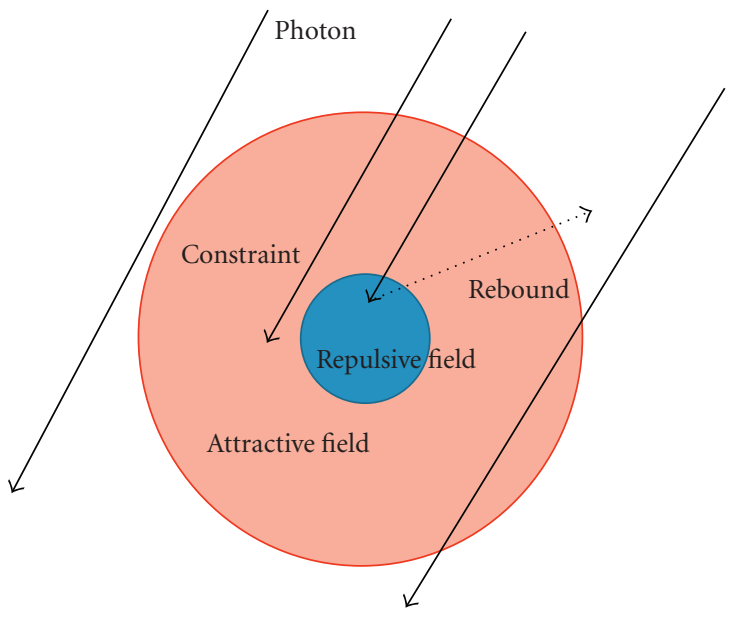

(a)

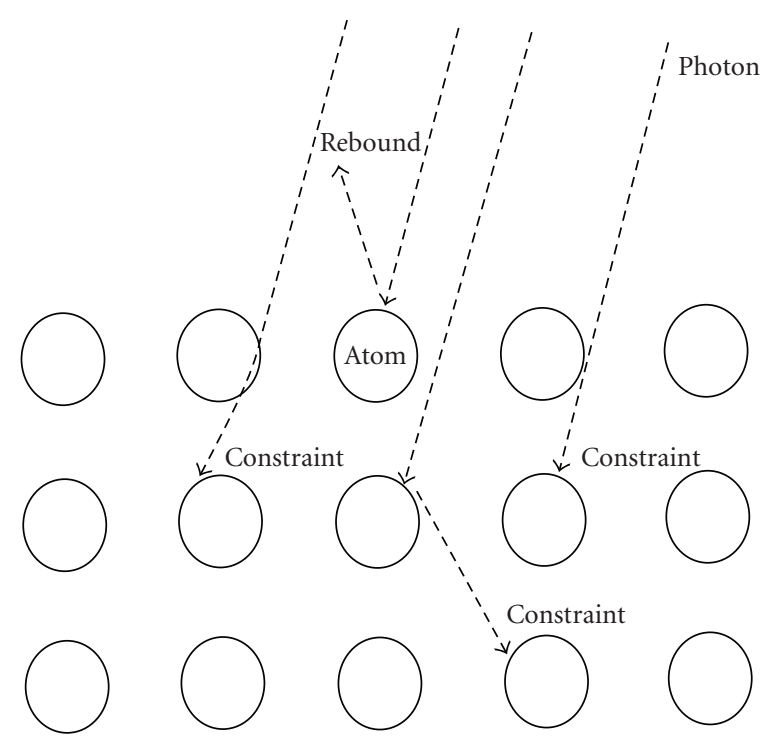

(b)

FIGURE 1: Illustrations of attractive and repulsive interactions of atoms and photons. (a) The photons moves toward repulsive force field are probably rebound out, and the other photons move through attractive field is constrained or decelerated. (b) The rebounded behavior is as same as reflection. The constraint behavior is as same as absorption.

each individual atom in the simulation domain since the Boltzmann distribution function allows the straightforward derivation of the mean kinetic energy $\left\langle E_{c}\right\rangle$ in the following way:

$$
\left\langle E_{c}\right\rangle=\frac{1}{2} M \sum_{i=1}^{N} v^{2}=\frac{3}{2} N k_{B} T_{\mathrm{MD}},
$$

where $k_{B}$ is the Boltzmann constant and $N$ is the number of particles in the system.

The initial temperature of the system was determined by Maxwell-Boltzmann distribution. During the indentation process, the velocity of every atom $v^{\text {new }}$ was scaled as

$$
v^{\text {new }}=v^{*} \sqrt{\frac{T_{D}}{T_{A}}},
$$

where $T_{D}$ is the desired temperature, $T_{A}$ is the actual temperature, and $v^{*}$ is the calculated velocity of atom. In this paper, the temperature was set as $300 \mathrm{~K}$.

Due to the actions of attractive and repulsive forces and the photon's energy dissipation, the velocity of a photon which enters the substrate deeply tends toward zero. This behavior is considered photon absorption in a solar cell. However, some photons are repulsed at the substrate surface due to the repulsive force. This is considered reflection. The photons whose velocity decreases to zero were removed to avoid the increasing of particle numbers, and then affect the system's energy.

Note that the efficiency of monocrystalline silicon is, at most, $24.7 \%$ [17]. This study attempts to make a model with an absorptance reaching to this theoretic value, $24.7 \%$ in a smooth nontextured substrate by simply adjusting $k$. In our simulation, $k$ is determined to be 3.86 in silicon. After this calculation, simulations of varying surface texturization with this potential model can proceed. The smooth surface model is a reference, and the other texturing models in this paper, which are simulated with the same conditions, can then be compared with the reference model. This methodology not only reasonably avoids the complexity of photonatom interaction but also achieves the goal of trying to evaluate the efficiency of two models with different surface texturization.

Following this concept, monocrystalline silicon was selected as the solar cell material. This is because silicon is the most common material in all types of solar cells, and its structure is simpler for using with a MD model. The results and conclusions of this study can also be extended to other solar cells, GaAs, InGaAs, InGaP, and so forth. The structure of monocrystalline silicon is an fcc diamond structure, showed in Figure 2. According the reviews of previous studies, square, pyramidal, and semicircular texturing structures were demonstrated. Under the texturing surface, three layers absorb the motion energy of photons. To avoid the effects of different lengths in the $x$ - and $y$-directions, the substrate of solar cells is set as a square. Periodic boundary conditions are imposed in the $x$ - and $y$-directions. Due to the periodic texturing shapes, different period lengths lead to different numbers of atoms. In the case of different intervals of square texturization, at least six periods are chosen. So in the case of distance $d=1$ cell, the number of silicon atoms is 144352. The longer the distance, the more atoms are present in the substrate. The photon's incident angle varies at $x$-zplanes.

All the simulations were executed with the computer of AMD Althlon64X2 3800+ CPU, Fedora Core Linux 6, and the GNU project FORTRAN compiler g77. 


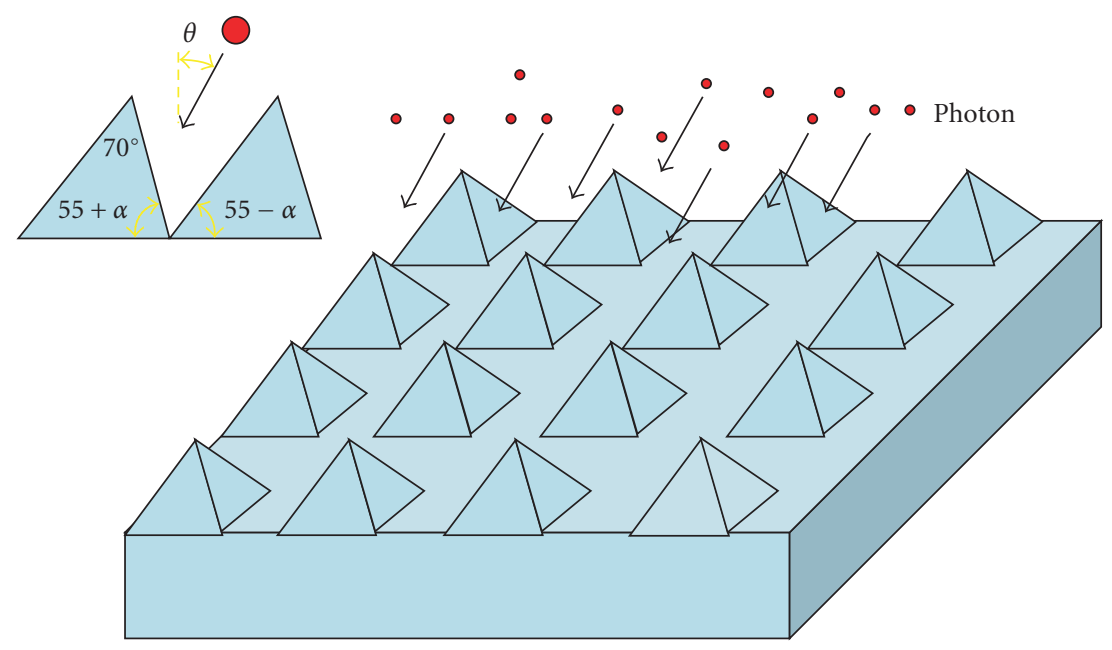

Figure 2: The model of mc-silicon by alkaline etching. The tilted angle is $\alpha$.

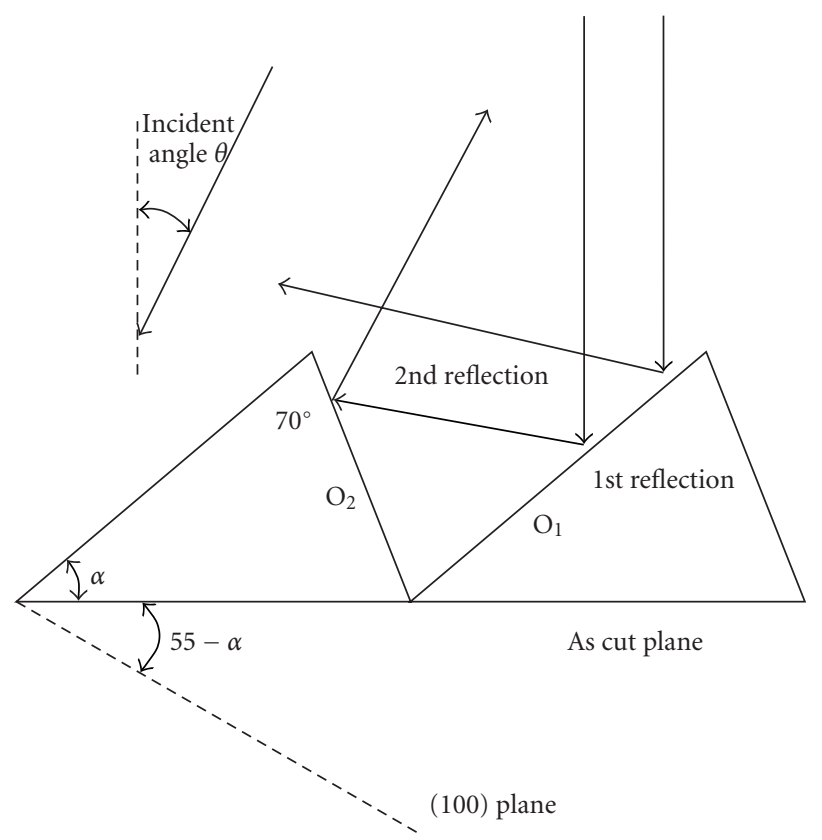

FIGURE 3: Schematic illustration of reflection on the surface of mcsilicon after alkaline etching.

\section{RESULTS AND DISCUSSION}

The variety of light absorptance of multicrystalline $\mathrm{Si}$ substrate etched by alkaline solutions is discussed in Figures $3-5$. According the discussion by Xi et al. [18], the tilted pyramids usually formed on the surface of mc-Si substrate etching by alkaline solution. If the orientation of the grains tends to (100), the pyramid will form a triangle with $70^{\circ}$ on the top, tilted angle $\alpha$ and $110^{\circ}-\alpha$ on the other angles. So, we try to make simulation with this model, and the results can easily compare with the results of Xi et al. [18].

Figure 4 shows that the reflection hardly changes when the tilted angle is less than $20^{\circ}$. That is because the most

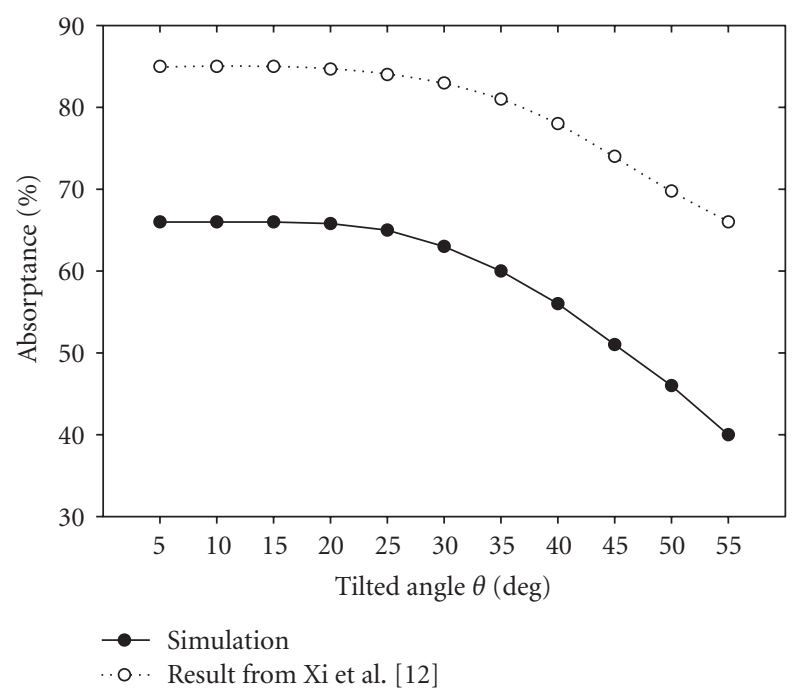

FIGURE 4: The relation of absorptance and the tilted angle, agreeing with the result of Xi et al. [18].

light reflects from $\mathrm{O}_{1}$ plane to $\mathrm{O}_{2}$ plane in Figures 3-5. More second reflection means that more light will be absorbed by the substrate. But if the tilted angle surpasses $20^{\circ}$, the reflectance increases quickly. Therefore, the pyramids with a large tilted angle lead to the lower efficiency. To compare with the results of Xi et al. [18], these two results are with a good agreement.

Furthermore, we try to change the incident angle $\theta$, shown in Figure 5. In the case of pyramid with tilted angle $\alpha=30^{\circ}$ (the three angles are $70^{\circ}, 80^{\circ}$, and $30^{\circ}$ ), more second reflection is produced when the incident angle $\theta=$ $20^{\circ} \sim 85^{\circ}$, so the absorptance rises higher to almost $60 \%$. But once the incident angle is greater than $80^{\circ}$, the possibility of second reflection decreased because the incident light is perpendicular to plane $\mathrm{O}_{2}$. Thus the absorptance quickly decreased. As the similar situation, when the incident angle is smaller than $-20^{\circ}$, the incident light is also perpendicular 


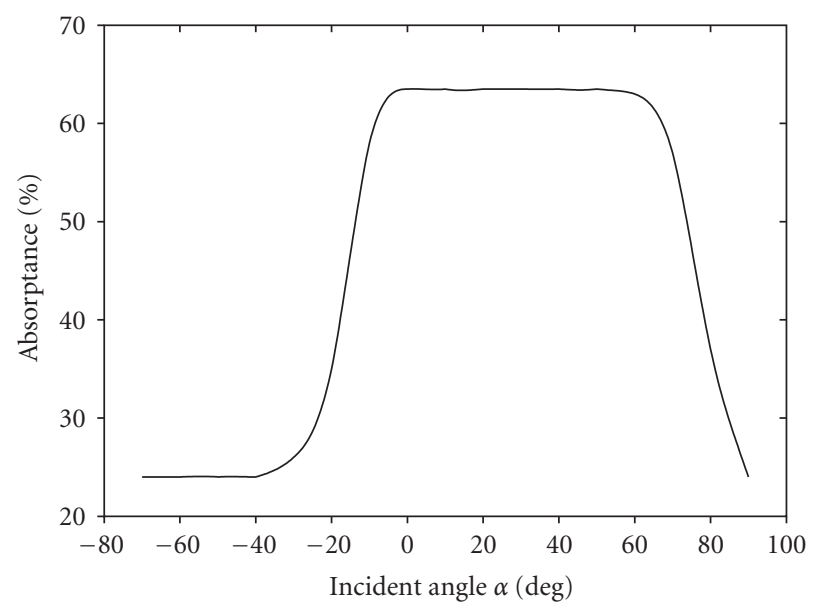

FIgURE 5: The relation of absorptance and incident angle.

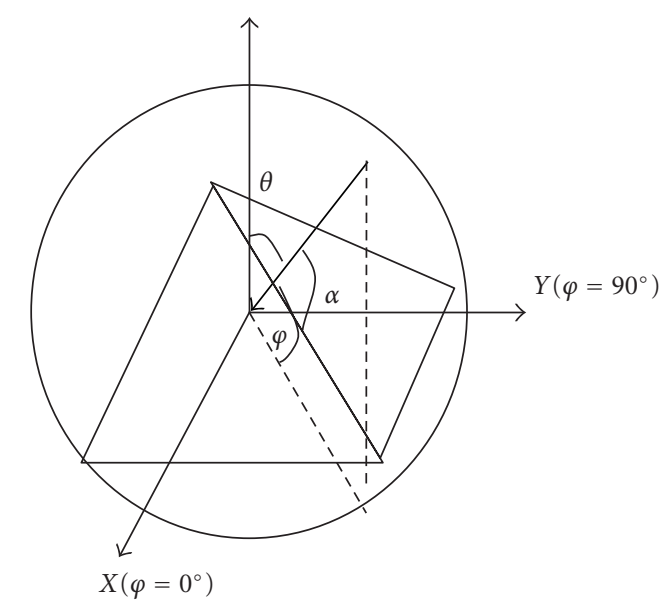

Figure 6: The diagram of a spherical polar coordinate system, which is quantified, is used to describe the surface morphology of the alkaline-etched wafers.

to plane $\mathrm{O}_{1}$, the possibility of second reflection decreased, so the absorptance decreased to almost $20 \%$.

We are interested about the effect of azimuth angle of incident light with absorptance. The surface morphology of the alkaline-etched wafers is quantified using a spherical polar coordinate system, as was the case for describing the relationship between different wafer and crystal orientations, shown in Figure 6. However, in this case, the tilt $(\alpha)$ and azimuth $(\varphi)$ angles refer to the positions of the normals to a particular facet of textured surface exposed by etch. These angles are measured relative to a particular crystal or wafer orientation, whose surface is parallel to the $x-z$ planes in the coordinate system and whose surface normal is parallel to the $Z$ axis. The positions of the etch facets formed on a particular crystal or wafer orientation are thus described in terms of their angles $\alpha$ and $\varphi$ with respect to the base orientation.

The simulation is proceeding with the conditions of the incident angle $\theta=30^{\circ}$, isosceles pyramid of tilted angle $\alpha=55^{\circ}$, and the azimuth angle $\varphi=0^{\circ}$ to $90^{\circ}$, to explore

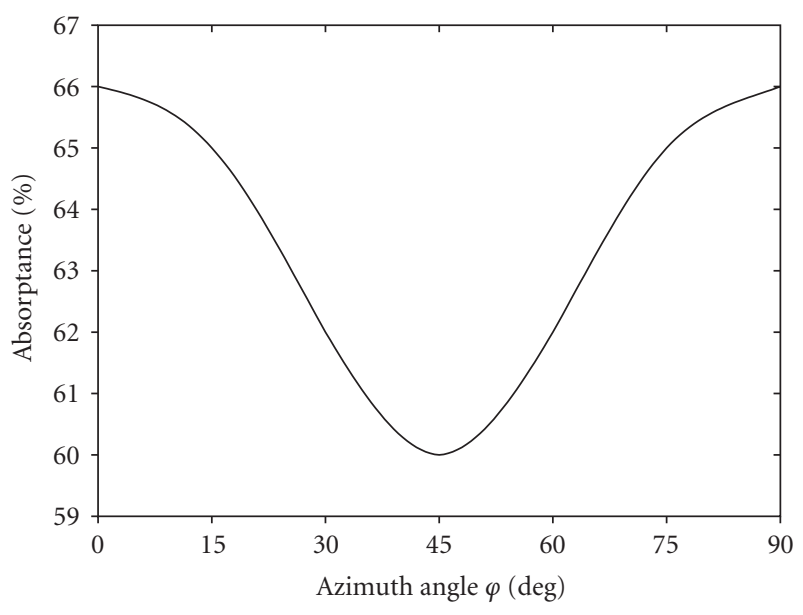

FIGURE 7: The position of the triangle of principal orientations. The incident angle $\theta$ and the azimuth angle $\varphi$ are demonstrated.

TABLe 1: Parameters of physical models, the bandgaps of the most important semiconductors for solar-cell applications.

\begin{tabular}{lc}
\hline \multicolumn{3}{c}{ Fundamental quantities } \\
\hline \multicolumn{3}{c}{ Multicrystalline silicon } \\
Mass (density) & $2.33 \mathrm{~g} / \mathrm{cm}^{3}$ \\
Length $\sigma$ & $5.431 \AA$ \\
Energy $\varepsilon$ & $1.792 \times 10^{-19} \mathrm{~J}$ \\
Time $t$ & $1.77 \times 10^{-3} \mathrm{ps} / \mathrm{step}$ \\
\hline \multicolumn{3}{c}{ Normalized quantities } \\
\hline Light speed (photon's speed) $c^{*}$ & $4.8 \times 10^{6}$ \\
Temperature $T^{*}$ & 0.0231 \\
Mass $m^{*}$ & 0.54 \\
\hline
\end{tabular}

the influence of azimuth angle on the absorptance of solar cells with pyramid surface. In Figure 7, it is found that the absorptance is the greatest when the azimuth angle $\varphi=0^{\circ}$ or $90^{\circ}$, the incident light is perpendicular to the (100) plane for the azimuth angle. The reflective light is usually reflected by planes of another pyramid. The higher probability of second reflection leads to the higher absorptance. But when the azimuth angle is getting larger, more incidents reflect only once, the probability of second reflection slightly decreased. Thus, the absorptance also decreased. When the azimuth angle is greater than $45^{\circ}$, the incident light illuminates the (010) plane. The absorptance slightly increases again.

\section{CONCLUSION}

In conclusion, this study proposes a new and simple MD model alternative to complex electron-photon interactions in quantum scale to analyze surface texturization of solar cells. Three surface texturization shapes are simulated with various angles of incident light. This methodology can easily determine the absorptance differences of various surface texturizations and suggest better texturization shapes. 
In the simulations of multicrystalline silicon by alkaline etching, the large tilted angle leads to the lower efficiency of solar cell. To consider the effect of incident angle, a range of high efficiency exists due to the increasing probability of second reflection. Furthermore, the azimuth angle of incident light also affects the efficiency of solar cells.

In this study, the wave behavior of photons and atoms is ignored. That means, according to the wave-particle duality, the gap between our study and real phenomenon possibly exists. However, this study is a beginning to simulate the absorption by molecular dynamics. Our results also agree well with previous studies. Thus, the development of this MD model is helpful to simulate the texturization before solar cells fabrication and can potentially be used to predict the efficiency promotion in any optical reflection-absorption cases.

\section{REFERENCES}

[1] J. D. Hylton, A. R. Burgers, and W. C. Sinke, "Alkaline etching for reflectance reduction in multicrystalline silicon solar cells," Journal of the Electrochemical Society, vol. 151, no. 6, pp. G408G427, 2004.

[2] Y. Nishimoto and K. Namba, "Investigation of texturization for crystalline silicon solar cells with sodium carbonate solutions," Solar Energy Materials and Solar Cells, vol. 61, no. 4, pp. 393-402, 2000.

[3] U. Gangopadhyay, K. H. Kim, S. K. Dhungel, et al., "A novel low cost texturization method for large area commercial mono-crystalline silicon solar cells," Solar Energy Materials and Solar Cells, vol. 90, no. 20, pp. 3557-3567, 2006.

[4] N. Marrero, B. González-Díaz, R. Guerrero-Lemus, D. Borchert, and C. Hernández-Rodríguez, "Optimization of sodium carbonate texturization on large-area crystalline silicon solar cells," Solar Energy Materials and Solar Cells, vol. 91, no. 20, pp. 1943-1947, 2007.

[5] Z. Xi, D. Yang, W. Dan, C. Jun, X. Li, and D. Que, "Investigation of texturization for monocrystalline silicon solar cells with different kinds of alkaline," Renewable Energy, vol. 29, no. 13, pp. 2101-2107, 2004.

[6] M. Lipinski, P. Panek, E. Bełtowska, and H. Czternastek, "Reduction of surface reflectivity by using double porous silicon layers," Materials Science and Engineering B, vol. 101, no. 1-3, pp. 297-299, 2003.

[7] D. H. Macdonald, A. Cuevas, M. J. Kerr, et al., "Texturing industrial multicrystalline silicon solar cells," Solar Energy, vol. 76, no. 1-3, pp. 277-283, 2004.

[8] J. Nijs, S. Sivoththaman, J. Szlufcik, et al., "Overview of solar cell technologies and results on high efficiency multicrystalline silicon substrates," Solar Energy Materials and Solar Cells, vol. 48, no. 1-4, pp. 199-217, 1997.

[9] J. Qian, S. Steegen, E. Vander Poorten, D. Reynaerts, and H. Van Brussel, "EDM texturing of multicrystalline silicon wafer and EFG ribbon for solar cell application," International Journal of Machine Tools and Manufacture, vol. 42, no. 15, pp. 1657-1664, 2002.

[10] W. A. Nositschka, C. Beneking, O. Voigt, and H. Kurz, "Texturisation of multicrystalline silicon wafers for solar cells by reactive ion etching through colloidal masks," Solar Energy Materials and Solar Cells, vol. 76, no. 2, pp. 155-166, 2003.

[11] M. Spiegel, C. Gerhards, F. Huster, W. Jooss, P. Fath, and E. Bucher, "Industrially attractive front contact formation methods for mechanically V-textured multicrystalline silicon solar cells," Solar Energy Materials and Solar Cells, vol. 74, no. 1-4, pp. 175-182, 2002.

[12] C.-F. Li, D. B. Johnson, and R. Kovacevic, "Modeling of waterjet guided laser grooving of silicon," International Journal of Machine Tools and Manufacture, vol. 43, no. 9, pp. 925-936, 2003.

[13] L. A. Dobrzanski and A. Drygala, "Laser texturisation of crystalline silicon for solar cells," in Proceedings of the 13th International Scientific Conference on Achievements in Mechanical and Materials Engineering (AMME '05), GliwiceZakopane, Poland, May 2005.

[14] M. M. D. Ramos and H. M. G. Correia, "Quantum modelling of photo-excited processes," Applied Surface Science, vol. 248, no. 1-4, pp. 450-454, 2005.

[15] W. Stier and O. V. Prezhdo, "Non-adiabatic molecular dynamics simulation of ultrafast solar cell electron transfer," Journal of Molecular Structure: THEOCHEM, vol. 630, no. 1-3, pp. 3343, 2003.

[16] J. H. Simmons, "What is so exciting about non-linear viscous flow in glass, molecular dynamics simulations of brittle fracture and semiconductor-glass quantum composites," Journal of Non-Crystalline Solids, vol. 239, no. 1-3, pp. 1-15, 1998.

[17] M. A. Green, K. Emery, D. L. King, Y. Hishikawa, and W. Warta, "Solar cell efficiency tables (version 29)," Progress in Photovoltaics, vol. 15, no. 1, pp. 35-40, 2007.

[18] Z. Xi, D. Yang, W. Dan, C. Jun, X. Li, and D. Que, "Texturization of cast multicrystalline silicon for solar cells," Semiconductor Science and Technology, vol. 19, no. 3, pp. 485489, 2004. 


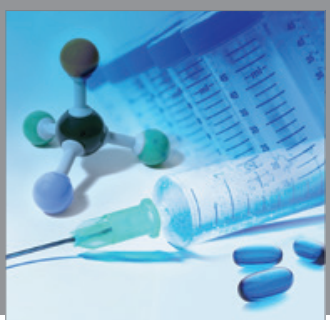

International Journal of

Medicinal Chemistry

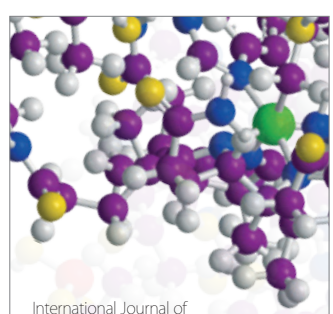

Carbohydrate Chemistry

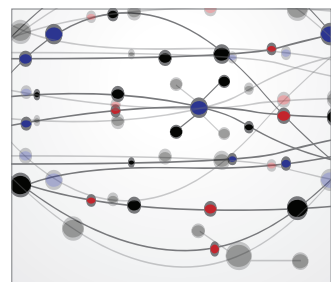

The Scientific World Journal
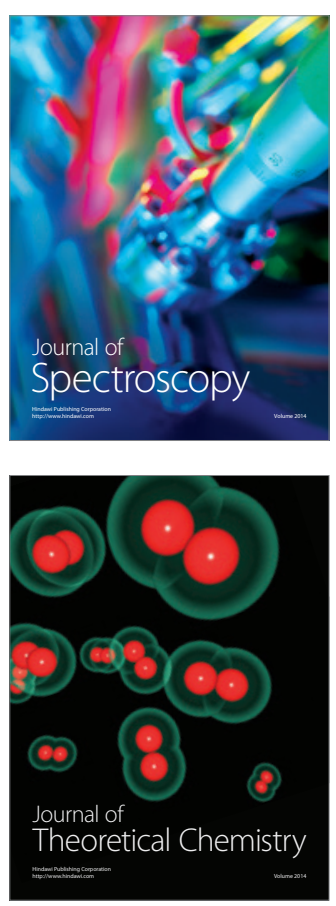
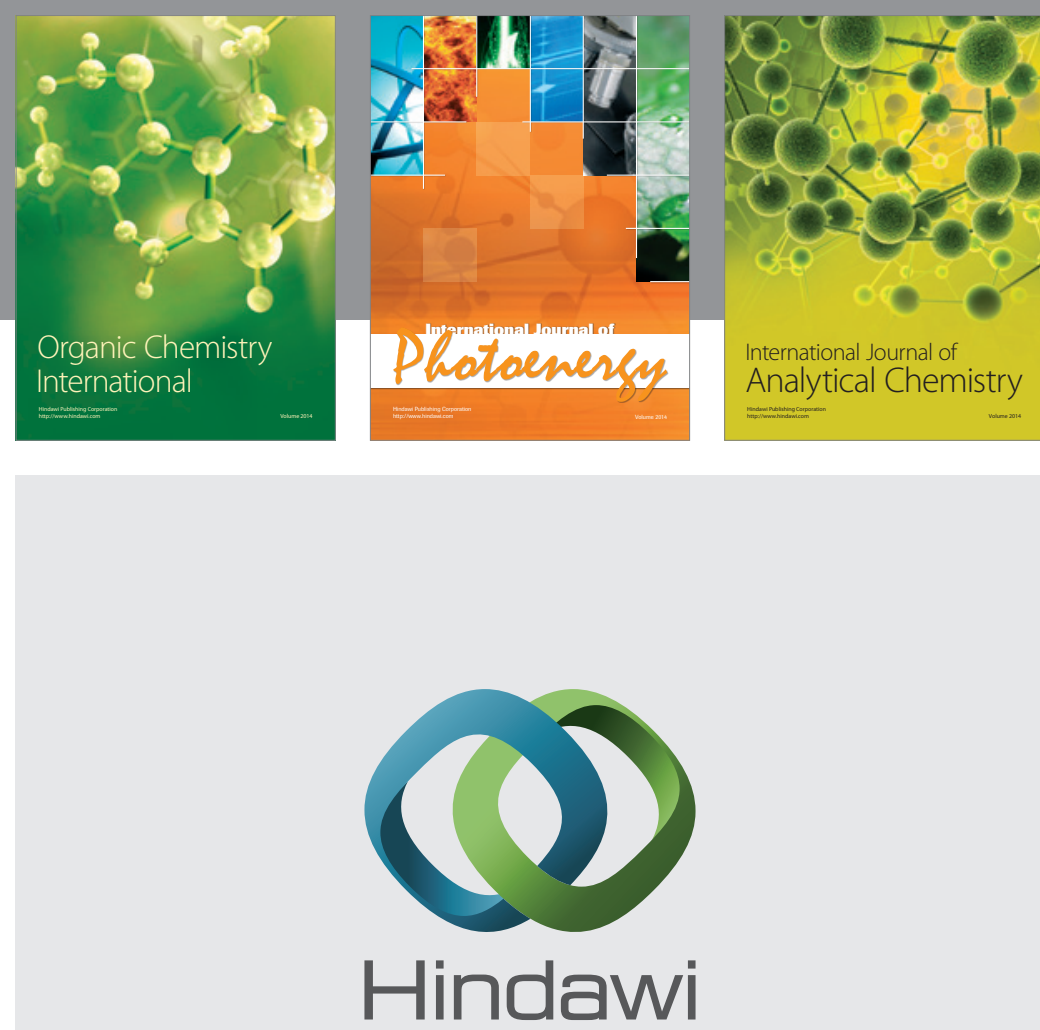

Submit your manuscripts at

http://www.hindawi.com
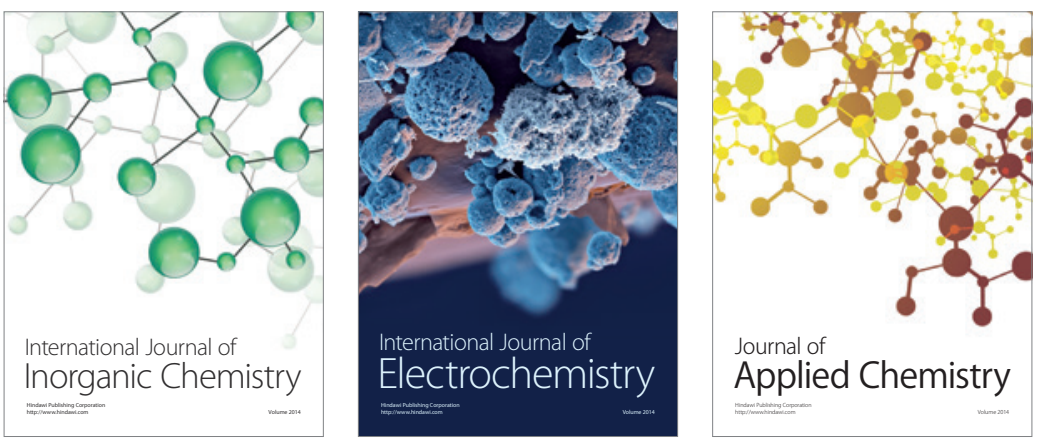

Journal of

Applied Chemistry
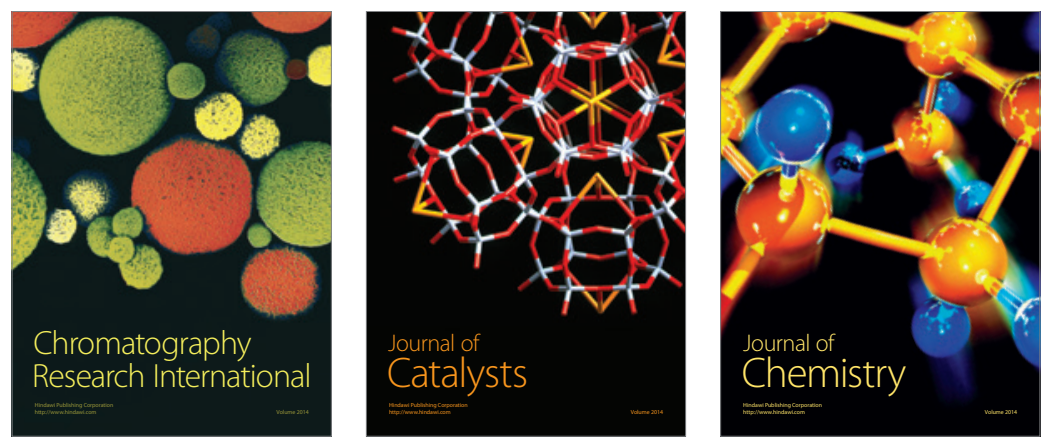
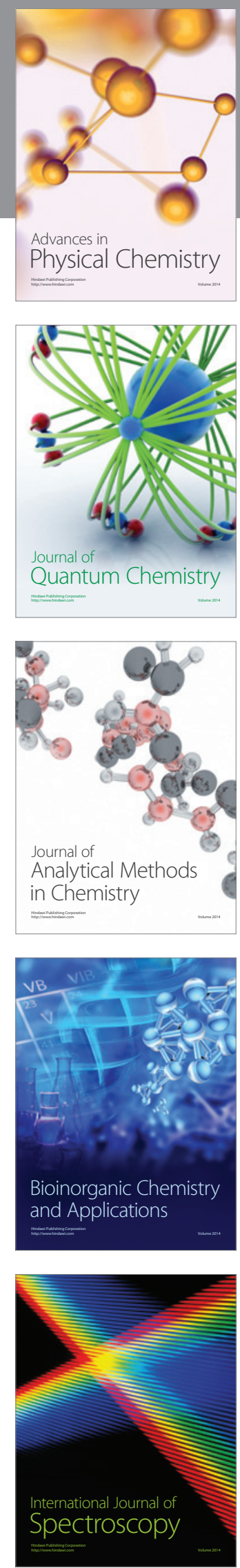